\title{
3D internal multiples prediction and attenuation workflow in data domain in the Santos Basin
}

Nathalia Ladeira Casado, Frederico Xavier de Melo, Samuel Madden, Marina Fagundes Pantoja, Elena Medina, Schlumberger

Copyright 2021, SBGf - Sociedade Brasileira de Geofísica

This paper was prepared for presentation during the $17^{\text {th }}$ International Congress of the Brazilian Geophysical Society held in Rio de Janeiro, Brazil, 16-19 August 2021.

Contents of this paper were reviewed by the Technical Committee of the $17^{\text {th }}$ International Congress of the Brazilian Geophysical Society and do not necessarily represent any position of the SBGf, its officers or members. Electronic reproduction or storage of any part of this paper for commercial purposes without the written consent of the Brazilian Geophysical Society is prohibited.

\begin{abstract}
Internal multiple reverberations in seismic data are commonly related to scattering reflection events originated by at least a single strong impedance contrast in the overburden, such as water bottom and/or salt boundaries. The complex geological setting in the Santos Basin generates internal multiples directly interfering with primary reflection imaging at deep reservoir levels. Prediction and subtraction of internal multiples in the data domain is a challenge given the subsurface complexity and overall coherency between predicted and observed multiples, which is a pertinent scenario in offshore Brazil. A common seismic processing strategy regionally adopted in the industry is to perform prediction in data domain, followed by model matching and subtraction in image domain. In this case study we adopted a different strategy where the subtraction was also implemented in data domain. The main motivation is to produce pre migrated dataset free of internal multiples suitable for data domain velocity analysis, model updates, and better conditioned for pre-stack inversion. Validation of the subtraction results performed in the imaging space as a quality control measure, showing comparisons before and after internal multiple attenuation, with additional seismic inversion related attributes, such as AVO analysis.
\end{abstract}

\section{Introduction}

Since the discovery of the pre-salt play in 2006, the Santos Basin has achieved global importance for the oil and gas industry. The exploration success is mainly due to the presence of permeable and porous carbonate reservoirs, salt seal and multiple traps (Buckley et. al, 2015). The pre salt active petroleum system is the Itapema-Itapema/Barra Velha (Moreira et. al, 2007). Source rocks are the lacustrine shales of the Itapema Formation. Pre-salt reservoirs rocks are formed by the Aptian microbial carbonates of Itapema and Barra Velha Formations. For this play the extensive and complex salt body are an efficient seal.

Internal multiple contamination in deep exploration plays is an intricate and challeging problem. This phenomenon negatively affects the quality of migrated data, compromising seismic interpretation, prestack inversion and reservoir characterization studies. Attenuation of these multiples while preserving primary reflection information can decrease uncertainty and ambiguity during model building and reservoir estimation. The Santos Basin geology is composed of complex stratified salt bodies with varying thickness, carbonates, igneous layers, sandstones, shales and evaporites. Attenuation of internal muliples are still not frequently applied in this region, even though its geological setting creates the perfect condition for the occurrence of these coherent noise events at the reservoir level.

Although all formations above the reservoir level are potential internal multiple generators, it is safe to assume that they should interact with at least one region in the subsurface with a strong impedance contrast. Assuming these formations act as an upward generator, we narrow down the contamination originating from regions around the salt boundaries (top and base), followed by remaining sequences up to the seafloor. It is possible to identify these generators and internal multiples associated with by forward modeling, pre-migration interpretation, well log data, as well as VSP analysis (Xavier de Melo et al., 2017).

The overall geological complexity present in the region calls for internal multiple prediction methods that require no or little prior information about the subsurface, with an acceptable compute cost when applied in large 3D seismic studies. Van der Neut and Wapenaar (2016) proposed a modification of the Marchenko algorithm to predict internal multiples with relatively good accuracy, requiring only a fictitious boundary positioned between the overburden and target formations. This method can predict several internal multiples simultaneously, so long the upward reflection of internal multiples sits above the pre-defined boundary. Compared with existing downward boundary- or layer-based (Wu et al., 2011, Xavier de Melo et al., 2020) and inverse-scattering series (Weglein et al. 1997) methods, the modified Marchenko-based algorithm provides an accurate and cost-effective solution when the reservoir or target of interest is clearly defined. Predicted models are then subtracted from the recorded seismic using adaptive matching schemes. Different matching methods and strategies are available, depending on the project objective and/or complexity of the predicted multiples.

In this case study we applied an internal multiple attenuation workflow based on the modified Marchenkobased prediction (Van de Neut and Wapenaar, 2016 and Wu et al., 2020) using a narrow azimuth (NAZ) towed streamer acquired over a deep-water region of the Santos Basin. The main reservoir is on the pre-salt level, below a thick stratified salt body, with strong internal multiples generated by carbonate sediments and salt. We defined 
three fictitious boundaries around the major upward generators, simultaneously predicting most of the internal multiples arriving at the zone of interest, followed by an adaptive matching method in the complex curvelet space. The proposed workflow performs the internal multiple operation in the data domain, validating the results and computing quality assurance attributes in the imaging space.

\section{Method}

Prior internal multiple attenuation, the recorded data must be free of random and coherent noise such anomalous amplitudes, ghost effect and surface related multiples. We achieved this requirement by applying a tailored preprocessing workflow consisting of low-frequency swell noise removal, adaptive deghosting and true-azimuth 3D surface multiple related elimination.

After the pre-processing, the internal multiple attenuation workflow consisted of the following steps:

- Identifying and understanding the internal multiple events and main generators;

- 3D data-driven internal multiple prediction;

- Adaptive matching in the data domain using a curvelet transform based method;

- Quality control and assurance in the data and imaging space.

The first step when trying to attenuate internal multiples is to visually identify its major occurrence in the seismic data. We were able to identify major internal multiples when inspecting limited angle/offset images along with selected common imaging point (CIP) gathers. Figure 1 shows one example where it is possible to visualize areas or focus contaminated by internal multiples.

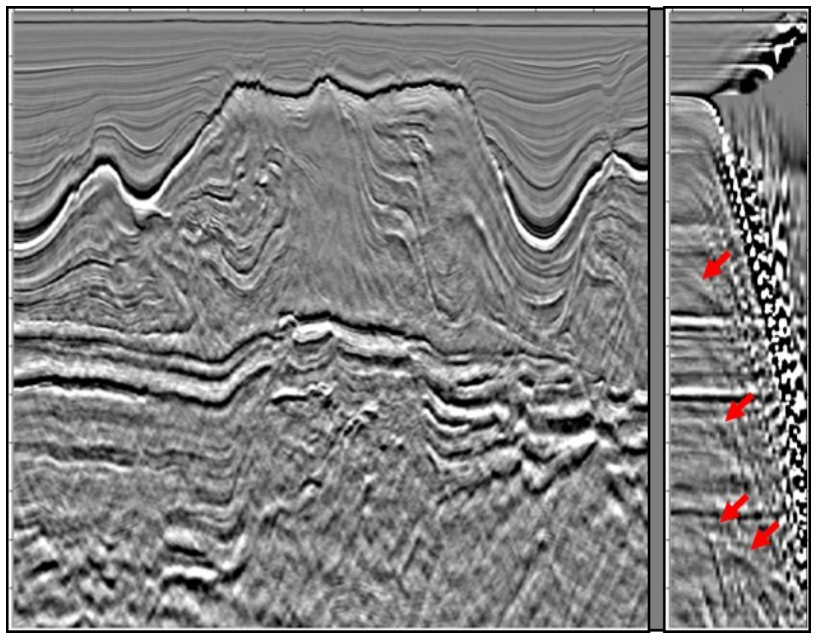

Figure 1 - Single offset $(1315 m$ - 1415m) migrated (PSDM) after surface related multiple attenuation (left) and a CIP gather from the same image. Red arrows show coherent and focused events that are potentially related as internal multiples. We use this visual inspection as way to trace back plausible places or formations responsible to generate internal multiples at the target zone.
After visually identifying major internal multiples in the seismic image, it is possible to infer places in the overburden consistent with occurrence of the multiple at the target level, either by spatial or temporal correlation. Based on the analysis from Figure 1, the following formations are likely associated with major upward generators of internal multiples: Top of Salt and Base of Salt. Figure 2 shows a common trace gather overlaid with major formations. Although salt boundaries are the major impedance boundaries, we notice significant contrast above the salt up to the seafloor acting as downward multiple generator. It is important to highlight that these subseafloor formations can also act as upward generators and should not be ignored when selecting the optimal boundaries required for the internal multiple prediction step.

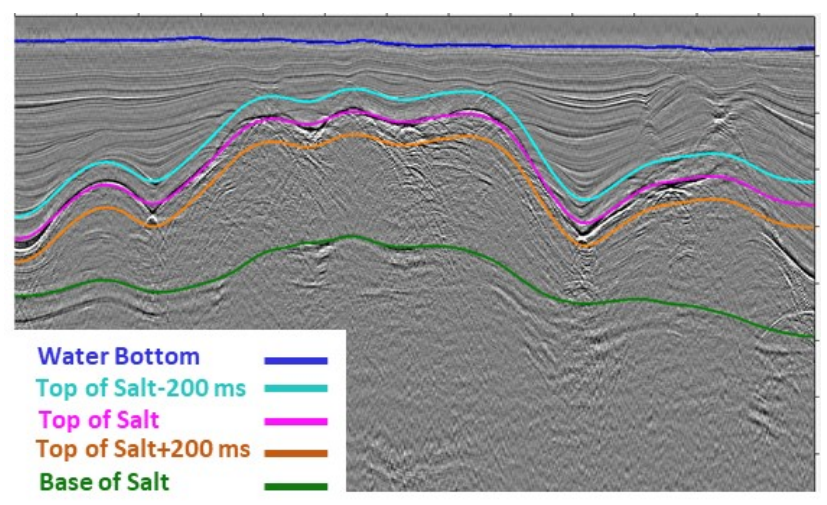

Figure 2 - Common Trace Gather (time domain) showing the main formations acting as major upward internal multiple generators: Top of Salt (purple) and Base of Salt (green). Light blue and orange horizons represent a pseudo-boundary definition designed to give proper weight to internal multiples solely generated above the salt and ones with salt formation included.

The data-driven method proposed by Van der Neut and Wapenaar (2016) is based on modified crosscorrelations followed by convolutions operations to construct an internal multiple model. This method requires a virtual boundary as input defining the region responsible for generating multiples (overburden) and the interval were internal multiples interfere with primaries (target).

The simple example illustrated in Figure 3 shows how the method works (Wu et al., 2020). For a four-layer model with a fictitious horizon $t_{2}$ separating the overburden from the target (Figure $3 a$ ), the prediction of the multiple $\boldsymbol{M}_{1}$ in equation 1 is given by a correlation of the data using muting operators $\theta$ between $t_{0}$ and $t_{2}$ (virtual event), followed its convolution with the data with a muting operator below $t_{2}$. This operation will predict all internal multiples generated above the boundary $t_{2}$, along with a single-sided internal multiple where one of its components has an upward reflection below t2. More details about the method can be found at Wu et al. (2020).

The Santos Basin case study required three fictitious boundaries covering different aspects of internal multiples generators: just above the Top of Salt, Intra-Salt and 

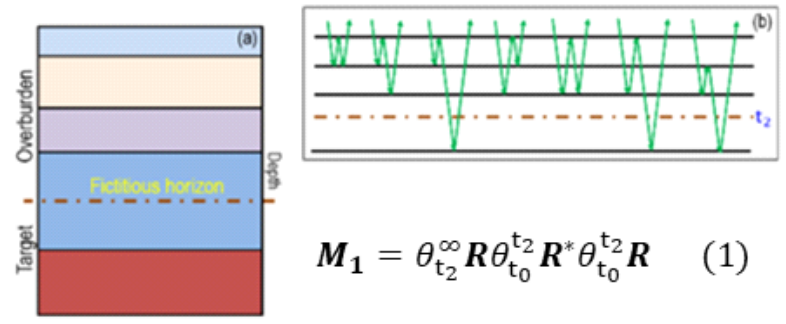

$M_{1}=\theta_{\mathrm{t}_{2}}^{\infty} R \theta_{\mathrm{t}_{0}}^{\mathrm{t}_{2}} \boldsymbol{R}^{*} \theta_{\mathrm{t}_{0}}^{\mathrm{t}_{2}} \boldsymbol{R}$

Figure 3 - Figure (a) illustrates a four-layer model with a fictitious horizon at the time $t_{2}$ separating the model into overburden and target. Illustration in (b) shows all possible internal multiples $\boldsymbol{M}_{\mathbf{1}}$ predictions based on the equation (1) (extracted from Wu et al., 2020).

around the Base of Salt (Figure 2). Choosing these boundaries allowed us to focus on a finite interval of internal multiples with different characteristics, such as relative amplitude and transmission absorption losses at the target level, not fully captured by the prediction process. Additionally, internal multiple models were constructed using an optimized aperture selection capturing the subsurface complexity in the region (Xavier de Melo et al., 2020). This process simultaneously predicts the internal multiples associated with these three boundaries, followed by a crosstalk separation process designed to remove overlapping information between models sharing a sequence of downward generation formations.

Attenuating predicted internal multiples from the observed seismic required a strategy that maximizes the separability between the observed coherent noise and primary reflections during the matching process. We performed the matching process in the curvelet domain by using cascaded and variable soft-thresholding of each predicted and adjusted model against the seismic data prior subtraction. The curvelet transform allowed internal multiples and primaries being mapped in a semi-sparse complex space, where splitting the wavefield into different dips and frequency scales maximized the separability between primaries and internal multiples.

Validation and quality assurance of the internal multiple attenuation used a combination of data and imaging domain displays and attributes. This is required when assessing the impact of the process in deep pre-salt reservoir levels. Imaging domain QC products used a Kirchhoff depth migration algorithm using a $50 \mathrm{~m} \times 50 \mathrm{~m}$ grid as reference.

\section{Results}

Initial validation of the internal multiple attenuation process is performed after the adaptive matching in the data space. Figure 4 shows a common mid-offset gather example (around $1800 \mathrm{~m}$ ) where the modeling and adaptive matching were able to attenuate strong internal multiple interference. We observed a significant coherent noise removal of strong and interpretable internal multiples, predominantly arriving in the intra-salt sequence, as highlighted by the white arrows.
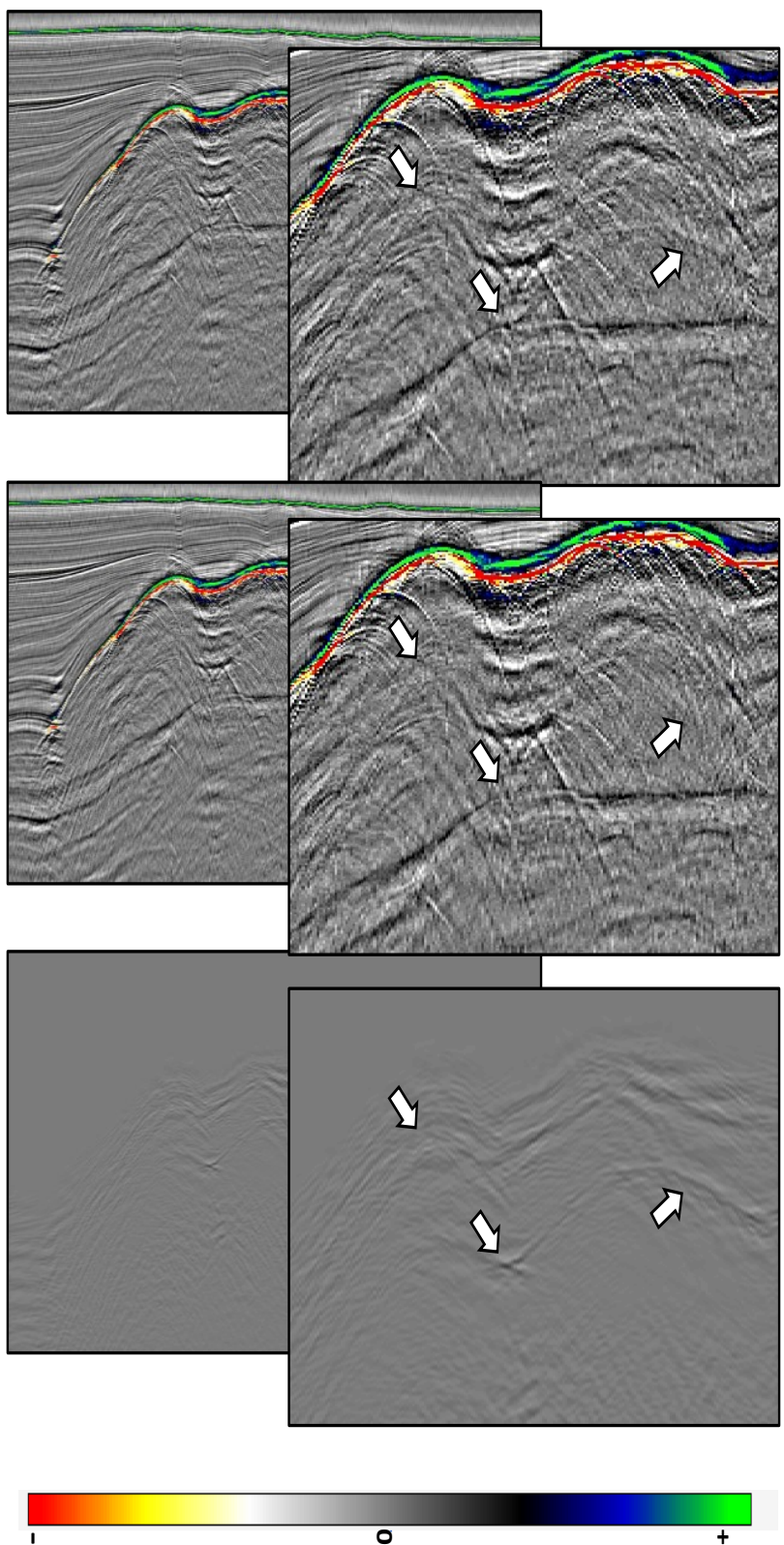

Figure 4 - Mid-offset (approximately $1800 \mathrm{~m}$ ) common channel gather before (top), after (middle) and difference (bottom) results of the internal multiple processing. Arrows highlight the attenuation of strong internal multiples in the intra-salt sequence.

Assessing and validating internal multiple attenuation results in deeper target formations are not straightforward tasks when performed in data domain due the overburden geological complexity, especially when dealing with a rugose a stratified salt interval. Imaging domain QC products provide an accurate inference regarding the seismic response when removing internal multiples. Figure 5 shows a Kirchhoff pre-stack image results over two distinct zones where we observe a subtle attenuation of internal multiples manifesting as crosstalk in the intra- 
salt (as also per Figure 4) and coherently interfering with pre-salt primaries.
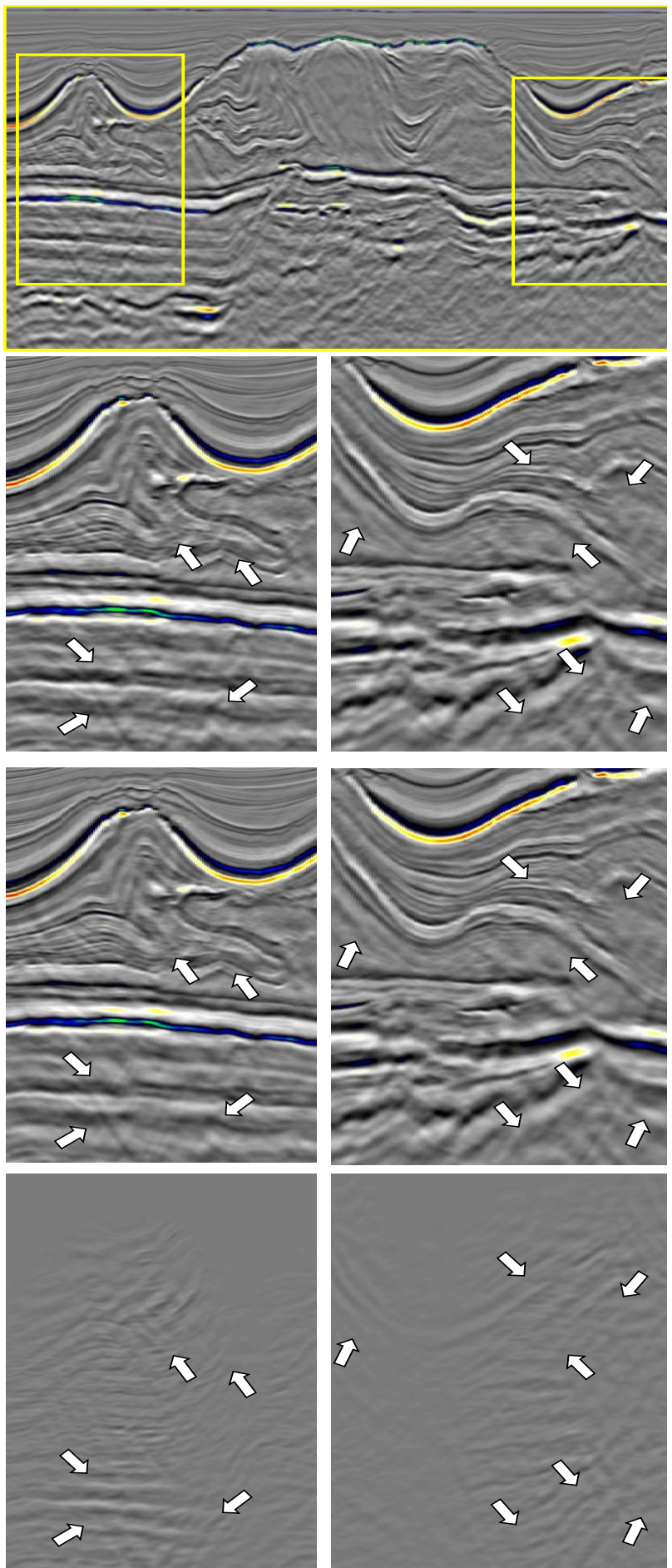

Figure 5 - Kirchhoff pre-stack migration with two highlighted zones (top) showing images before (second from the top), after (middle) and difference (bottom) results of the internal multiple processing. Arrows highlight the attenuation of strong internal multiples in the intra-salt and pre-salt sequences.
A closer inspection performed across CIP gathers in Figure 6 shows a clearer response of the proposed workflow, with visible attenuation of internal multiples with little or no velocity discrimination with underlying primaries. This type of interference can be pervasive, as it directly interferes with the true AVO response expected in the target pre-salt intervals.

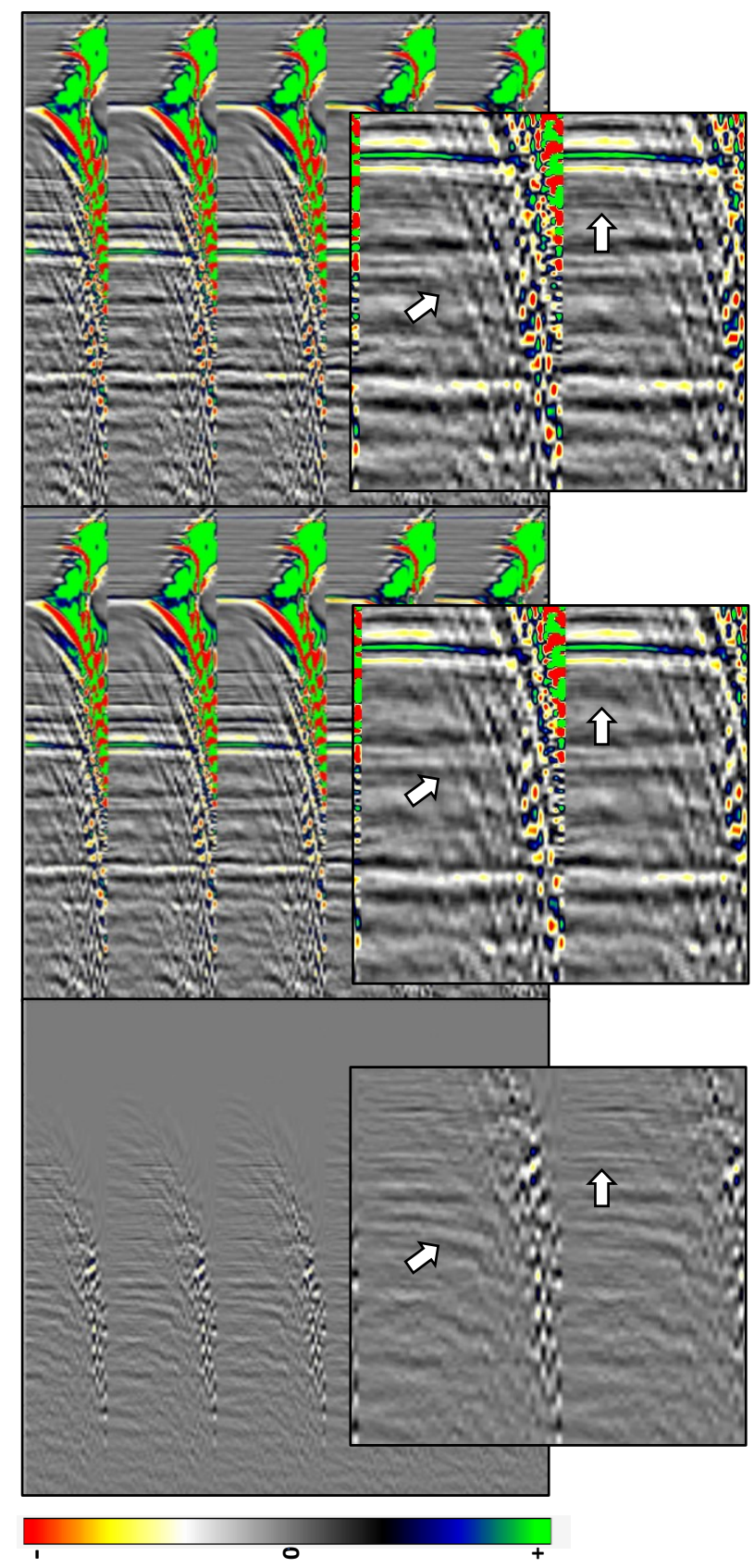

Figure 6 - CIP gathers showing the seismic data before (top), after (middle) and estimated internal multiples (bottom). White arrows inside the detailed display show the positive impact of the attenuation of complex internal multiples events, including those with little or no velocity discrimination from underlying primaries. 


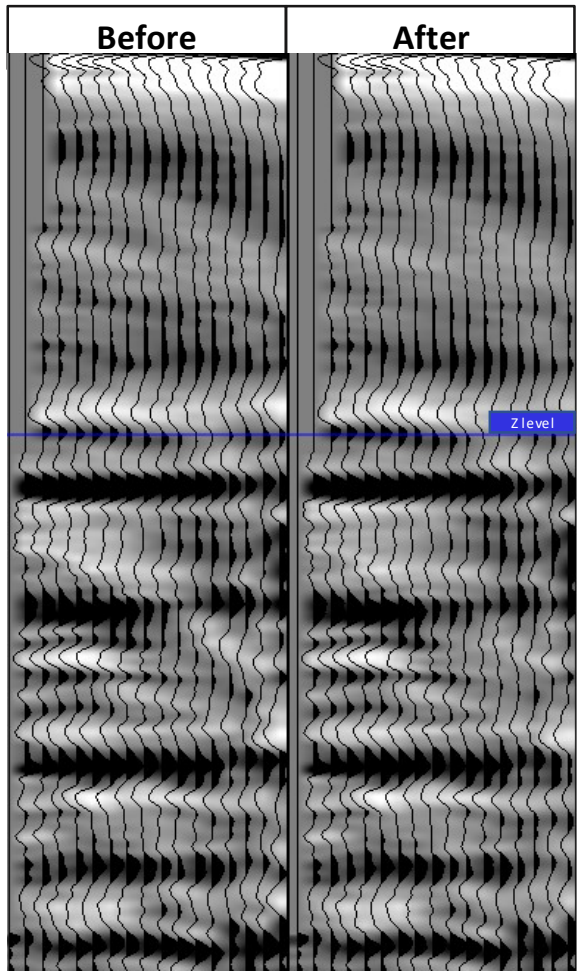

口8_XIMP Coefficient of determination $r^{2}=0.076$

AFT_XIMP Coefficient of determination $r^{2}=0.290$

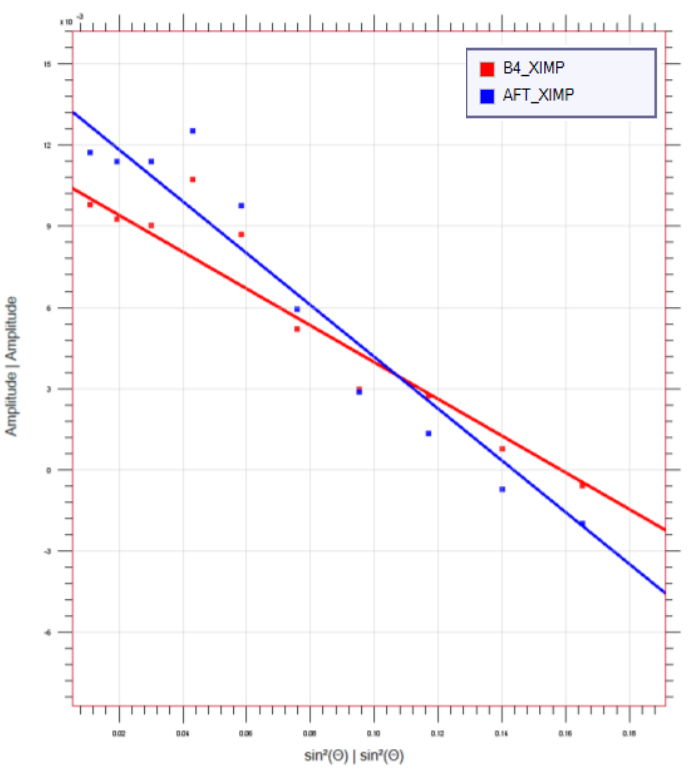

Figure 7 - Amplitude versus offset analysis of a common image point before and after internal multiple attenuation. The crossplot between the seismic amplitude (vertical axis) versus the squared sine (horizontal axis) of the incident angle shows a better fit when performing the linear regression after the internal multiple attenuation (AFT_XIMP) with a higher coefficient of determination.
The interference (constructive or destructive) in the seismic data varies according to the angle of incidence of internal multiples and primaries. The former is usually a result of several short- and long-period scattering effects, resulting in focused events overlapping with primary reflections. These effects significantly change with offset and/or angle of incidence, being predominately stronger at the near/narrow and rapidly decaying moving towards far/wider ranges. This is normally the case for $P$-wave reflection events and internal multiples scattered from within, converted waves and its internal multiples behave differently and are beyond the scope of this work.

Figure 7 illustrates this behavior when extracting AVO attributes from the seismic CIP gather. A closer examination over an event at a given $Z$ level shows an amplitude versus the square sine of the angle of incidence comparison before and after the application of the proposed internal multiple attenuation workflow. Although the visual comparison from the seismic data is subtle, the crossplot shows less amplitude variability at the near angle of incidence after applying the internal multiple attenuation process. Linear regressions performed in both cases show a better fit when extracting attributes from the seismic data after the internal multiple attenuation, shown by a significantly higher coefficient of determination $\mathrm{r}^{2}$.

\section{Conclusions}

This case study showed an internal multiple attenuation strategy designed to improve the primary response situated at the main reservoir sequence on the pre-salt level. We defined three fictitious boundaries around the major upward generators, simultaneously predicting most of the internal multiples arriving at the zone of interest, followed by an adaptive matching method in the complex curvelet space. The proposed workflow performed the whole internal multiple operation in the data domain, with additional validation of the results in the imaging space.

Visual inspection of the seismic data showed a clear reduction of internal multiples, especially in places where there was a clear discrimination from primaries, such as events arriving within the salt region. In places where the contamination was mainly constructive with no clear discrimination between primaries and internal multiples, such as the pre-salt targets, AVO attribute extraction showed the positive impact of the proposed internal multiples attenuation workflow. Quantitative metrics extracted from the seismic data showed a more stable amplitude variation with angle of incidence after attenuating internal multiples.

\section{References}

Buckley, J. P., Boscence, D. \& Elders, C., 2015. Tectonic setting and stratigraphic architecture of an Early Cretaceous lacustrine carbonate platform, Sugar Loaf High, Santos Basin. Geological Society, London, Special Publications, pp. 175-191. 
Moreira, J. L. P., Madeira, C. V., Gil, J. A. \& Machado, M. A. P., 2007. Bacia de Santos. Boletim Geociencias Petrobras, maio/nov, Volume 15, pp. 531-549.

Van der Neut, J., and K. Wapenaar, 2016, Adaptive overburden elimination with the multidimensional Marchenko equation, Geophysics, 81, T265-T284.

Weglein, A.B., F.A. Gasparotto, P.M. Carvalho, and R.H. Stolt, 1997, An inverse-scattering series method for attenuating multiples in seismic reflection data, Geophysics, 62, 1975-1989.

Wu J., Wu Z., de Melo F. X., Lapilli C. M., and Kostov C., 2020 , Internal multiples prediction with separation of overburden and target applied to a shallow-water streamer dataset, SEG Technical Program Expanded Abstracts : 3174-3178.

Wu, Z. J., S. Sonika, and W. Dragoset, 2011, Robust Internal Multiple Prediction Algorithm: 81st Annual International Meeting, SEG, Expanded Abstracts, 35463549.

Xavier de Melo, F., A. Glushchenko, G. Nyein, W. Krissat, C. Kostov, K.M. Al Hosani, L. Gerardo Figuera, M. Aamir, A. Cooke, 2017, Improving Inversion Accuracy by Optimal Internal Multiple Attenuation - A Case Study from the UAE. 79th EAGE Conference \& Exhibition, Extended Abstracts.

Xavier de Melo, F., M.F. Pantoja, M. Ortin, E. Medina, 2020, Optimized 3D and true-azimuth internal multiple attenuation strategy in the Santos Basin, SEG Technical Program Expanded Abstracts 2020, 1052-3812. 